Proceedings of the XV-th National School "Hundred Years of Superconductivity", Kazimierz Dolny, October 9-13, 2011

\title{
On the Imbalanced d-Wave Superfluids Within the Spin Polarized Extended Hubbard Model: Weak Coupling Limit
}

\author{
A. KuJAWA-CICHY* \\ Solid State Theory Division, Faculty of Physics, Adam Mickiewicz University \\ Umultowska 85, 61-614 Poznań, Poland
}

\begin{abstract}
We investigate the superfluid properties of d-wave pairing symmetry within the Extended Hubbard Model $(\mathrm{EHM})$ in a magnetic field. We analyze the temperature and magnetic field dependencies of the order parameter. We find that in the two-dimensional case, the spatially homogeneous spin polarized superfluidity $(S C(P \neq 0))$ is stable in the weak coupling limit, at $T=0$, as opposed to the s-wave pairing symmetry case in $2 \mathrm{D}$. We construct the ground state phase diagrams both for fixed chemical potential and electron concentration. Furthermore, we obtain the temperature vs. magnetic field and temperature vs. spin polarization phase diagrams.
\end{abstract}

PACS: 71.10.Fd, 74.20.Rp, 71.27.+a, 71.10.Hf

\section{Introduction}

In this paper, we briefly discuss the superfluid properties of the Extended Hubbard Model (EHM) with spin independent hopping integrals $\left(t^{\uparrow}=t^{\downarrow}\right)$, in a Zeeman magnetic field $(h)$. We take into account only the pure d-wave pairing symmetry case. Our motivation to study this kind of pairing symmetry is not only the interest in high-temperature superconductivity, but also the possibility of existence of new phases with non-trivial Cooper pairing mechanism in imbalanced Fermi gases.

There has been much experimental [1] and theoretical [2-8] work on the possibilty of existence of the spatially homogeneous spin-polarized superfluidity (Sarma phase or breached pairing state (BP)) with one or two Fermi surfaces (BP-I or BP-II, respectively) and a gapless spectrum for the majority spin species. The coexistence of the superfluid and the normal component in the isotropic state is characteristic for the BP phase. According to many investigations $[6,8]$, at $T=0$, the Sarma phase (or BP-II state) in the weak coupling limit is unstable for the s-wave pairing symmetry case.

The model Hamiltonian is the Extended Hubbard model (EHM) [9] in a magnetic field with spin independent hopping integrals:

$$
\begin{aligned}
\hat{H} & =\sum_{i, j, \sigma}\left(t_{i j}-\mu \delta_{i j}\right) \hat{c}_{i \sigma}^{\dagger} \hat{c}_{j \sigma}+U \sum_{i} \hat{n}_{i \uparrow} \hat{n}_{i \downarrow} \\
& +\frac{1}{2} \sum_{i, j, \sigma, \sigma^{\prime}} W_{i j} \hat{n}_{i \sigma} \hat{n}_{j \sigma^{\prime}}-h \sum_{i}\left(\hat{n}_{i \uparrow}-\hat{n}_{i \downarrow}\right),
\end{aligned}
$$

where: $t_{i j}$ - nearest-neighbor hopping; $\sigma=\uparrow, \downarrow-$ spin index, $\hat{n}_{i \sigma}=\hat{c}_{i \sigma}^{\dagger} \hat{c}_{i \sigma}$ - particle number operator, $U$ on-site interaction, $W_{i j}$ - intersite interaction, $h$ - Zeeman magnetic field, $\mu-$ chemical potential. The gap parameter is defined by: $\Delta_{\boldsymbol{k}}=\frac{1}{N} \sum_{\boldsymbol{q}} V_{\boldsymbol{k} \boldsymbol{q}}^{\boldsymbol{s}}\left\langle\hat{c}_{-\boldsymbol{q} \downarrow} \hat{c}_{\boldsymbol{q} \uparrow}\right\rangle$, where: $V_{\boldsymbol{k} \boldsymbol{q}}^{s}=-U-W \gamma_{\boldsymbol{k}-\boldsymbol{q}}$.
Applying the broken symmetry Hartree-Fock approximation, we obtain the grand canonical potential $\Omega$ and the free energy $F$. Using the free energy expression, one gets the equations for the gap: $\Delta_{\boldsymbol{k}}=\frac{1}{N} \sum_{\boldsymbol{q}} V_{\boldsymbol{k} \boldsymbol{q}}^{s} \frac{\Delta_{\boldsymbol{q}}}{2 \omega_{\boldsymbol{q}}}\left(1-f\left(E_{\boldsymbol{q} \uparrow}\right)-f\left(E_{\boldsymbol{q} \downarrow}\right)\right)$, particle number (which determines $\mu$ ): $n=n_{\uparrow}+n_{\downarrow}, n_{\sigma}=$ $\frac{1}{N} \sum_{\boldsymbol{k}}\left\langle\hat{c}_{\boldsymbol{k} \sigma}^{\dagger} \hat{c}_{\boldsymbol{k} \sigma}\right\rangle=\frac{1}{N} \sum_{\boldsymbol{k}}\left(\left|u_{\boldsymbol{k}}\right|^{2} f\left(E_{\boldsymbol{k} \sigma}\right)+\left|v_{\boldsymbol{k}}\right|^{2} f\left(-E_{\boldsymbol{k}-\sigma}\right)\right)$, Fock parameter: $p=\frac{p_{\uparrow}+p_{\downarrow}}{2}, p_{\sigma}=\frac{1}{N} \sum_{\boldsymbol{k}} \gamma_{\boldsymbol{k}}\left\langle\hat{c}_{\boldsymbol{k} \sigma}^{\dagger} \hat{c}_{\boldsymbol{k} \sigma}\right\rangle=$ $\frac{1}{N} \sum_{\boldsymbol{k}} \gamma_{\boldsymbol{k}}\left(\left|u_{\boldsymbol{k}}\right|^{2} f\left(E_{\boldsymbol{k} \sigma}\right)+\left|v_{\boldsymbol{k}}\right|^{2} f\left(-E_{\boldsymbol{k}-\sigma}\right)\right)$ and spin magnetization: $M=n_{\uparrow}-n_{\downarrow}$, where: $f\left(E_{\boldsymbol{k} \sigma}\right)=1 /\left(\exp \left(\beta E_{\boldsymbol{k} \sigma}\right)+\right.$ $1), \beta=1 / k_{B} T, E_{\boldsymbol{k} \downarrow, \uparrow}= \pm \frac{U M}{2} \pm \frac{1}{2} W\left(p_{\uparrow}-p_{\downarrow}\right) \frac{\gamma_{\boldsymbol{k}}}{\gamma_{0}} \pm h+\omega_{\boldsymbol{k}}$, $\omega_{\boldsymbol{k}}=\sqrt{\left(\left(\epsilon_{\boldsymbol{k}}-\bar{\mu}\right)^{2}+\left|\Delta_{\boldsymbol{k}}\right|^{2}\right.},\left|v_{\boldsymbol{k}}\right|^{2}=\frac{1}{2}\left(1-\frac{\epsilon_{\boldsymbol{k}}-\bar{\mu}}{\omega_{\boldsymbol{k}}}\right),\left|u_{\boldsymbol{k}}\right|^{2}=$ $1-\left|v_{\boldsymbol{k}}\right|^{2}, \epsilon_{\boldsymbol{k}}=-2 t \Theta_{\boldsymbol{k}}, \gamma_{\boldsymbol{k}}=2 \Theta_{\boldsymbol{k}}, \Theta_{\boldsymbol{k}}=\sum_{l=1}^{d} \cos \left(k_{l} a_{l}\right)$ ( $d=2$ for two-dimensional lattice), $a_{l}=1$ in further considerations, $\bar{\mu}=\mu-n\left(\frac{U}{2}+W \gamma_{0}\right)$.

We take into account only the pure d-wave pairing symmetry case. Then, $W<0, U=0$ and the equation for the order parameter takes the form:

$$
\frac{4}{|W|}=\frac{1}{N} \sum_{\boldsymbol{k}} \frac{\eta_{\boldsymbol{k}}^{2}}{2 \omega_{\boldsymbol{k}}}\left(1-f\left(E_{\boldsymbol{k} \uparrow}\right)-f\left(E_{\boldsymbol{k} \downarrow}\right)\right),
$$

where: $\eta_{\boldsymbol{k}}=2\left(\cos k_{x}-\cos k_{y}\right)$.

We also calculate the superfluid density $\rho_{s}(T)$, which takes the form:

$$
\begin{aligned}
& \rho_{s}(T)=-\frac{t}{N} \sum_{\boldsymbol{k}}\left(\frac{\epsilon_{\boldsymbol{k}}-\bar{\mu}}{2 \omega_{\boldsymbol{k}}} \cos k_{x} X_{\boldsymbol{k}}\right. \\
& \left.+t \sin ^{2} k_{x} Y_{\boldsymbol{k}}\right),
\end{aligned}
$$

where:

$$
X_{\boldsymbol{k}}=\frac{\sinh \left(\beta \omega_{\boldsymbol{k}}\right)}{\cosh \left(\beta\left(h+\frac{U M}{2}\right)\right)+\cosh \left(\beta \omega_{\boldsymbol{k}}\right)},
$$




$$
Y_{\boldsymbol{k}}=\beta \frac{\cosh \left(\beta\left(h+\frac{U M}{2}\right)\right) \cosh \left(\beta \omega_{\boldsymbol{k}}\right)+1}{\left(\cosh \left(\beta\left(h+\frac{U M}{2}\right)\right)+\cosh \left(\beta \omega_{\boldsymbol{k}}\right)\right)^{2}} .
$$

The Kosterlitz-Thouless temperature $\left(T_{c}^{K T}\right)$ is determined in $d=2$ from the universal relation:

$$
k_{B} T_{c}^{K T}=\frac{\pi}{2} \rho_{s}\left(T_{c}^{K T}\right)
$$

\section{Numerical results}

One of the most important quantities related to superconductivity is the gap parameter. As is well known, the BCS theory predicts the existence of an isotropic order parameter, which vanishes at the temperature of the superconductor-normal phase transition. However, intensive studies of the gap parameter for high- $T_{c}$ superconductors indicate significant differences with respect to the predictions of the BCS theory. Most of the measurements show that its value in the ground state is much larger than the value of $\Delta$ in conventional superconductors [10]. The symmetry of the energy gap can be determined from measurements of the changes in its magnitude for different momentum directions $\left|\Delta_{k}\right|$. Most studies indicate the $d_{x^{2}-y^{2}}$ pairing symmetry (with the energy gap $\left.\Delta_{k}=\Delta_{\eta}\left(\cos \left(k_{x}\right)-\cos \left(k_{y}\right)\right)\right)$ [11].

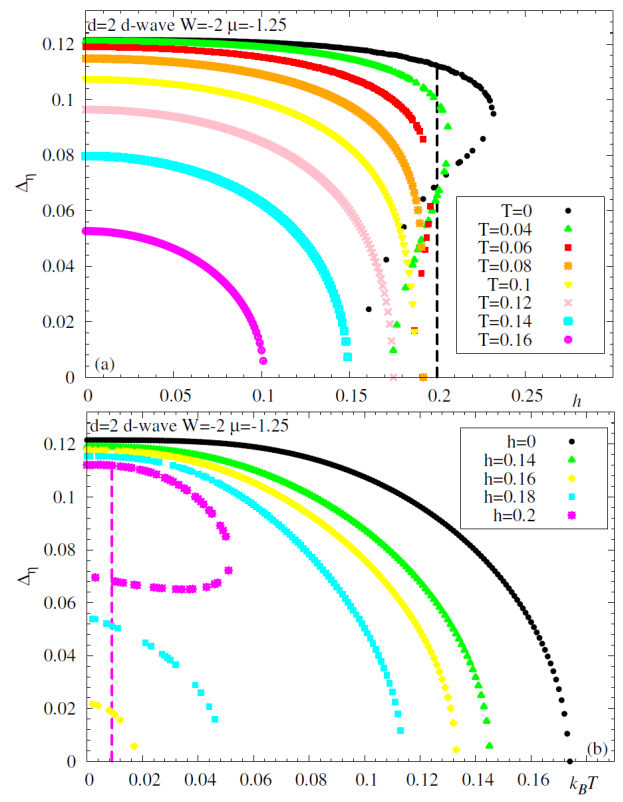

Fig. 1. Dependence of $\Delta_{\eta}$ on the magnetic field (a) and temperature (b), $W=-2$ for a fixed $\mu=-1.25$. In the pannel (b), for $h=0.16$ and $h=0.18$ the lower branches are unstable. For $T=0$ (a) and $h=0.2$ (b) the vertical dashed lines denote the first order phase transition from the magnetized superconductor state $(\operatorname{SC}(P \neq 0))$ to the normal state (NO).

The d-wave pairing symmetry is also very interesting from the point of view of the BP state (or Sarma phase) in imbalanced ultracold Fermi gases.
We start from the analysis of the influence of the magnetic field on the order parameter characteristics.

If $h=0$, in the case of the d-wave pairing symmetry, the order parameter vanishes for some values of the wave vector $\boldsymbol{k}$, i.e. along the lines $\left|k_{x}\right|=\left|k_{y}\right|$ (in four nodal points on the Fermi surface). Disappearance of the gap on the Fermi surface leads to the existence of zero energy quasiparticles. It is justified to believe that the Sarmatype phase will be stable at $h \neq 0$, in the weak coupling limit, as opposed to the s-wave pairing symmetry case in $2 \mathrm{D}$.

Figure 1 shows the dependence of the order parameter amplitude $\left(\Delta_{\eta}\right)$ on the magnetic field (a) and temperature (b), for $W=-2, \mu=-1.25$. As one can see in Fig. 1a, there are two different branches of the solutions for $\Delta_{\eta} \neq 0$, at $T=0$, as in the s-wave pairing symmetry case [12]. However, as opposed to the isotropic order parameter case, the upper branch of the solutions of $\Delta_{\eta}$ is dependent on the magnetic field in the ground state. Therefore, a finite polarization $(P=M / n)$ occurs in the system, for arbitrarily small value of the magnetic field, even at $T=0$. This is explained by the creation of polarized quasiparticle excitations in the nodal points of the gap $[13,14]$. Moreover, this branch is stable up to $h \approx 0.2$. At this point, the first order phase transition from the polarized superconducting to the normal state occurs. On the other hand, the lower branch, which also depends on $h$, is unstable at $T=0$. Obviously, the polarization increases with increasing temperature. Thus, the range of occurrence of the Sarma-type phase (the superconducting state with $P \neq 0$ ) increases.

As mentioned above, the d-wave pairing symmetry at $h=0$ is gapless in four nodal points on the Fermi surface. In the weak coupling limit, at $h=0$, the nodal points are located at $\left( \pm \frac{\pi}{2}, \pm \frac{\pi}{2}\right)$. However, the gap $\left(E_{g}\right)$ in the density of states is fixed by the location of the logarithmic singularities. The value of $E_{g}$ is determined by the maximum value of the energy gap: $E_{g}=2 \Delta_{\max }$, where $\Delta_{\max }=4 \Delta_{\eta}$.

The influence of the Zeeman magnetic field on the density of states is significant. If $h \neq 0$, the densities of states are different for the particles with spin "up" and spin "down". The gap appears in the density of states for the minority spin species and equals $2 h$ [15]. The occurrence of the gap in the density of states is caused by the existence of some minimum non-zero quasiparticle energy.

Now, let us consider the ground state phase diagrams for the d-wave pairing symmetry case, in the weak coupling regime. Both the fixed chemical potential and the fixed electron concentration case have been analyzed.

In the s-wave case and in the weak coupling regime, the superconducting phase with $P=0\left(\mathrm{SC}_{0}\right)$ is stable at $T=0$. However, the analysis of the $\mathrm{d}$-wave order parameter behavior, the density of states and the momentum distributions characteristics [15] indicate the possibility of the occurrence of stable $\mathrm{SC}(P \neq 0)$ phase at $T=0$, even in the weak coupling regime, as opposed to the s- 


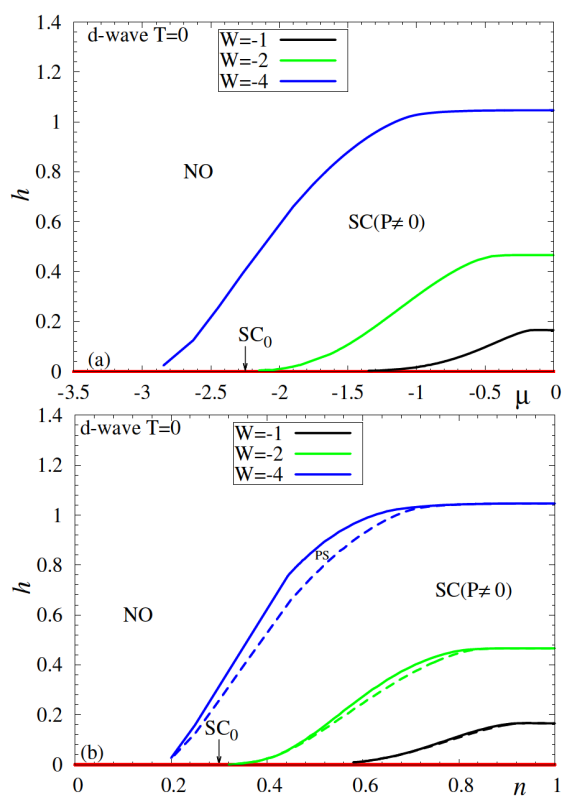

Fig. 2. Critical magnetic field vs. the chemical potential (a) and the electron concentration (b) for the first order $\mathrm{SC}(P \neq 0)$-NO transition, at $T=0$; three different values of the attractive interaction.

wave pairing symmetry case in $2 \mathrm{D}[16]$. As shown above, for infinitesimally low value of the magnetic field, the state is stable. The $\operatorname{SC}(P \neq 0)$ phase is the superfluid state of coexisting Cooper pairs and excess fermions, with the latter responsible for finite polarization (magnetization) and the gapless excitations characteristic for this state.

At higher values of the Zeeman magnetic field, $\mathrm{SC}(P \neq$ $0)$ is destroyed by the paramagnetic effect or by population imbalance. Then, there is the first order phase transition from the polarized superconducting phase to the polarized normal state. The first order transition is manifested by the presence of the phase separation (PS) region in the phase diagrams at fixed $n$ (see: Fig. $2 \mathrm{~b}$ ). The phase separation occurs between $\operatorname{SC}(P \neq 0)$ with the number of particles $n_{s}$ and NO with the number of particles $n_{n}$. It is worth mentioning that the d-wave superfluidity is stable around the half-filled band in the weak coupling limit and its range of occurrence widens with increasing attractive interaction.

Let us discuss the finite temperature phase diagrams.

One of the well-known results concerning the influence of the Zeeman magnetic field on superconductivity is the existence of the so-called Clogston limit [17]. In the weak coupling regime, for the s-wave pairing symmetry case, at $T=0$, the superconductivity is destroyed through the paramagnetic effect and the first order phase transition to the normal state at a universal value of the critical magnetic field $h_{c}=\Delta_{0} / \sqrt{2} \approx 0.707 \Delta_{0}$, where $\Delta_{0}$ is, the gap at $T=0$ and $h=0$. In turn, this universal value of the magnetic field in which the superconducting state is destroyed in the ground state, for the d-wave pairing sym-

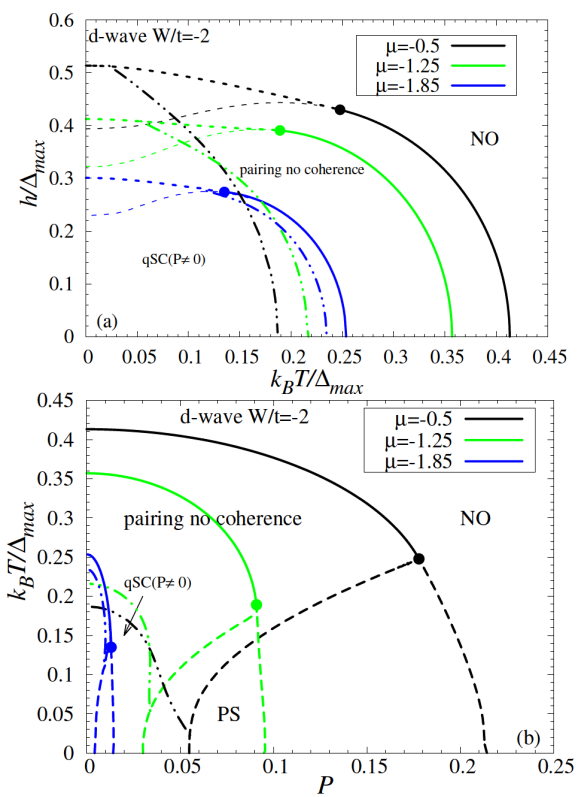

Fig. 3. Temperature vs. magnetic field (a) and polarization (b) phase diagrams for $W=-2$, three values of $\mu ; \mathrm{SC}_{0}-$ non-polarized superconducting state $(P=0)$, $\mathrm{SC}(P \neq 0)-2 \mathrm{D}$ superconductor in the presence of the polarization, NO - partially-polarized normal state. The thick solid line is the second order phase transition line from pairing without coherence region to NO. The thin dashed line is an extension of the 2nd order transition line (metastable solutions). The thick dasheddouble dotted line is the Kosterlitz-Thouless transition line. The thick dotted line denotes the first order phase transition to NO. $\Delta_{0}=4 \Delta_{\eta}$ denotes the gap at $T=0$ and $h=0$

metry case at half-filing is: $h_{c}^{d-w a v e}=0.56 \Delta_{\max }[14]$, where $\Delta_{\max }=4 \Delta_{\eta}$ at $T=0$ and $h=0$.

Figure 3 shows the temperature vs. magnetic field ( $T-$ $h$ ) and polarization $(T-P)$ phase diagrams for $W=-2$ and three values of the chemical potential. These fixed values of $\mu$ correspond to lower values of $n$ than $n=$ 1 , therefore the $\mathrm{d}$-wave Clogston limit is not reached. However, our results for $\mu=0(n=1)$ agree with the ones from the paper [14], i.e. indeed $h_{c}^{d-\text { wave }}=0.56 \Delta_{\max }$ for this case.

We take into account the phase fluctuations in $d=2$ within the KT scenario, as in the s-wave pairing symmetry case [8]. In such way, we can estimate the phase coherence temperatures, in addition to the mean field (MF) temperatures. The solid lines (2nd order transition lines) and the PS region are obtained within the mean field approximation (MFA). The curves below the first order phase transition lines on the phase diagrams (the thin dotted lines) are the extension of the 2nd order transition lines below tricritical points. The thick dash-double dotted lines denote the KT transition. The system is a quasi superconductor (qSC) below $T_{c}^{K T}$. Between $T_{c}^{K T}$ and $T_{c}^{H F}$ pairs still exist, but without a long-range phase coherence (the pseudogap behavior). The KT tempera- 
tures are much smaller than $T_{c}^{H F}$. This can be seen particularly clearly for fixed $\mu=-0.5$ and $W=-2$ case the difference between $T_{c}^{K T}$ and $T_{c}^{H F}$ amounts to nearly $50 \%$. However, this difference decreases with decreasing $\mu$ (decreasing $n$ ) and decreasing attraction (in the weak coupling limit). The second order phase transition takes place from the pairing without coherence region to the normal state at sufficiently low values of the magnetic field. With increasing $h$, the character of the transition between the pairing without coherence region and the normal state changes from the second to the first order, which is manifested by the existence of the MF tricritical point on the phase diagrams. The topology of the $(T-h)$ diagrams is the same as in the s-wave case [12].

However, qualitative differences between the s-wave and d-wave pairing symmetries are clearly visible in $(P-T)$ phase diagrams. At $T \geq 0$ and $P=0$, there is the unpolarized superconducting phase, both for the swave and the d-wave pairing symmetry case. At $T=0$, there is only the PS region, for the whole range of polarizations, i.e. $P>0$, for the isotropic order parameter case. In turn, in the d-wave pairing symmetry case, there is the spin-polarized superconducting phase at $T=0$, up to some critical value of the polarization, for which the first order phase transition to the normal state takes place. In the PS region, not only the polarizations, but also the particle densities in SC and NO are different. At $T=0$ and for the s-wave pairing symmetry, this separation region is between the $\mathrm{SC}_{0}$ phase and the normal state, while in the d-wave pairing symmetry it is between $\mathrm{SC}(P \neq 0)$ and NO. At $T \neq 0, \Delta \neq 0$ and $P \neq 0$, the system is also in the polarized qSC phase (i.e. homogeneous superconductivity in the presence of the spin polarization) in the s-wave pairing symmetry case up to $T_{c}^{K T}$. The KT phase is restricted to the weak coupling region and low values of $P$, as in the d-wave pairing symmetry case. Increasing polarization favors the phase of incoherent pairs. As shown in Fig. 3b, the range of occurrence of qSC in the presence of $P$ widens in the weak coupling regime with increasing $\mu$ (increasing $\mathrm{n}$ ). In the s-wave pairing symmetry case, one can distinguish the gapless region at sufficiently high values of the magnetic field and temperature. As mentioned before, the d-wave pairing symmetry at $h=0$ is gapless in four nodal points on the Fermi surface. Therefore, the natural consequence of this is the occurrence of the gapless region also for infinitesimally low values of $h$, even at $T=0$.

\section{Conclusions}

We have investigated the influence of a Zeeman magnetic field on the superfluid characteristics of the EHM, within the mean field approximation. We have analyzed the pure $d$-wave pairing symmetry case. At $T=0$, in the presence of the magnetic field, the ground state is the spatially homogeneous spin-polarized superfluid state, which has a gapless spectrum for the majority spin species, for a weak attraction, as opposed to the s-wave pairing symmetry case in $2 \mathrm{D}$. With increasing $h$, the first order phase transition takes place to the NO state. We have also ex- tended our analysis to finite temperatures in $d=2$ by invoking the KT scenario. At finite temperatures, in the weak coupling regime and for fixed $\mu$, the following states have been found in the $2 \mathrm{D}$ system: at $h=0$ - the $\mathrm{SC}_{0}$ phase; at $T=0, h \neq 0$ - polarized superfluid state with a gapless spectrum for the majority spin species; at $T>0-\mathrm{qSC}(P \neq 0)$ (below the Kosterlitz-Thouless temperature); region of pairs without coherence (below the Hartree temperature); the PS region and NO. PS terminates at MF TCP, in $(T-P)$ phase diagrams.

\section{Acknowledgments}

I would like to thank R. Micnas for guidance and many valuable discussions. I acknowledge financial support under grant No. N N202 030540 (MSHE - Poland).

\section{References}

[1] M.W. Zwierlein, A. Schirotzek, C.H. Schunck, W. Ketterle, Science 311, 495 (2006); G.B. Partridge, W. Li, R.I. Kamar, Y. Liao, R.G. Hulet, Science 311, 503 (2006).

[2] G. Sarma, J. Phys. Chem. Solids 24, 1029 (1963).

[3] D.E. Sheehy, L. Radzihovsky, Phys. Rev. Lett. 96, 060401 (2006).

[4] W.V. Liu, F. Wilczek, P. Zoller, Phys. Rev. A 70, 033603 (2004).

[5] M. Iskin, C.A.R. Sá de Melo, Phys. Rev. Lett. 97, 100404 (2006).

[6] M.M. Parish, F.M. Marchetti, A. Lamacraft, B.D. Simons, Phys. Rev. Lett. 98, 160402 (2007); M.M. Parish, F.M. Marchetti, A. Lamacraft, B.D. Simons, Nat. Phys. 3, 124 (2007).

[7] A. Kujawa-Cichy, Acta Phys. Pol. A 118, 423 (2010).

[8] A. Kujawa-Cichy, R. Micnas, Europhys. Lett. 95, 37003 (2011).

[9] R. Micnas, J. Ranninger, S. Robaszkiewicz, Rev. Mod. Phys. 62, 113 (1990); R. Micnas, B. Tobijaszewska, J. Phys. Cond. Matter 14, 9631 (2002).

[10] M.B. Maple, cond-mat/9802202 (1998).

[11] Z.-X. Shen, D.S. Dessau, B.O. Wells, D.M. King, W.E. Spicer, A.J. Arko, D. Marshall, L.W. Lombardo, A. Kapitulnik, P. Dickinson, S. Doniach, J. DiCarlo, T. Loeser, C.H. Park, Phys. Rev. Lett. 70, 1553 (1993); D.A. Bonn, Nat. Phys. 2, 159 (2006); J. Annett, N. Goldenfeld, A.J. Leggett, Physical Properties of High Temperature Superconductors, Vol. 5, World Scientific, Singapore 1996.

[12] A. Kujawa, R. Micnas, Acta Phys. Pol. A 114, 43 (2008).

[13] J.S. Tempere, N. Klimin, J.T. Devreese, V.V. Moshchalkov, Phys. Rev. B 77, 134502 (2008).

[14] K. Yang, S.L. Sondhi, Phys. Rev. B 57, 8566 (1998).

[15] A. Kujawa-Cichy, R. Micnas, in preparation.

[16] A. Kujawa, R. Micnas, Acta Phys. Pol. A 115, 138 (2009).

[17] A.M. Clogston, Phys. Rev. Lett. 9, 266 (1962). 